\title{
Recovery of miR-139-5p in Ovarian Cancer Reverses Cisplatin Resistance by Targeting C-Jun
}

\author{
Ying Jiang Jing Jiang Haiqing Jia Zhiwei Qiao Jingru Zhang \\ Department of Gynaecology, Cancer Hospital of China Medical University, Liaoning Cancer Hospital \& \\ Institute, Shenyang, China
}

\section{Key Words}

miR-139-5p • C-Jun • Cisplatin • Ovarian cancer

\begin{abstract}
Background/Aims: In platinum-based chemotherapy for ovarian cancer, acquired drug resistance is a frequent occurrence. Because recent studies have demonstrated that dysregulation of microRNAs (miRNAs) is partly responsible for the induction of acquired drug resistance in cancers, we hypothesized that correcting the dysregulation of key miRNAs would reverse the acquired resistance to platinum-based drugs in ovarian cancer. Methods: Cisplatinresistant SKOV3 and A2780 ovarian cancer cell lines (SKOV3-R and A2780-R, respectively) were established by long-term exposure to cisplatin. MTT assays were performed to evaluate the viability of SKOV3, SKOV3-R, A2780, and A2780-R cells. Quantitative PCR was used to examine the expression of miR-139-5p in these cell lines. The regulatory mechanism was confirmed by western blot analysis and luciferase reporter assays. After treatment with miR-139-5p and cisplatin, mitochondrial membrane potential and apoptosis were measured by using flow cytometry. Interaction with c-Jun and activating transcription factor 2 (ATF2) was evaluated by co-immunoprecipitation. Expression of B-cell lymphoma-extra large (Bcl-xl) and activation of caspase- 9 and caspase- 3 were detected by western blotting. Results: Expression of miR$139-5 p$ was decreased in SKOV3-R and A2780-R cells. Recovery of miR-139-5p increased the sensitivity of SKOV3-R and A2780-R cells to cisplatin treatment, inhibited the interaction of c-Jun and ATF2, and decreased Bcl-xl expression in SKOV3-R and A2780-R cells. Expression of miR-139-5p promoted cisplatin-induced mitochondrial apoptosis through binding the $3^{\prime}$ untranslated region of c-Jun mRNA. Conclusion: Recovery of miR-139-5p suppressed the expression of c-Jun and thus reversed cisplatin-resistance in ovarian cancer.
\end{abstract}




\section{Cellular Physiology Cell Physiol Biochem 2018:51:129-141 and Biochemistry \begin{tabular}{l|l} 
DOI: 10.1159/000495169 & (c) 2018 The Author(s). Published by S. Karger AG, Basel \\
www.karger.com/cpb
\end{tabular}}

Jiang et al.: miR-139 Reverses Cisplatin Resistance in Ovarian Cancer

\section{Introduction}

Ovarian cancer is the most lethal malignancy of the female genital system. Despite current developments in medical technology, the overall prognosis remains poor $[1,2]$. At present, surgery and chemoradiotherapy are the main treatment strategies for ovarian cancer. Although surgery is the most effective clinical strategy, it is not optimal for patients with advanced disease. Instead, systematic chemotherapy is usually used for this patient population [3, 4]. However, a large proportion of patients with ovarian cancer have a poor prognosis because of resistance to chemotherapy [5]. Thus, there is an urgent need to develop drugs that can overcome chemoresistance.

Cisplatin is the most commonly used platinum-based chemotherapeutic agent. Its antitumor activity is broad-spectrum and non-cell cycle-specific, because its mechanism of action is to crosslink DNA, subsequently inhibiting DNA replication and transcription and inducing apoptosis in tumor cells [6, 7]. Despite the fact that cisplatin is an effective therapeutic agent for ovarian cancer, the development of chemoresistance has become a major obstacle to its widespread clinical use [8,9]. Thus, there is a need to develop effective strategies for preventing or reversing cisplatin resistance in this disease.

MicroRNAs (miRNAs) are a class of small and non-coding RNAs with a length of 18-25 nucleotides [10]. They can bind to the $3^{\prime}$ untranslated region ( $3^{\prime}$ UTR) of target mRNAs to form an RNA-induced silencing complex, inducing the translational repression and degradation of their target mRNAs. Because approximately $60 \%$ of human genes are regulated by miRNAs, they regulate nearly every cellular process including cell proliferation, differentiation, metastasis, metabolism, and apoptosis. However, miRNA expression profile is frequently dysregulated in cancers $[11,12]$. Moreover, the dysregulation of miRNA expression is reportedly responsible for the development of chemoresistance in multiple cancers including ovarian cancer. Therefore, correcting miRNA dysregulation may be a potential strategy for reversing chemoresistance [13-17].

In this study, we investigated the role of miR-139-5p in the development of cisplatin resistance in ovarian cancer.

\section{Materials and Methods}

\section{Cell lines}

The human ovarian cancer cell lines SKOV3 and A2780 were purchased from the Institute of Biochemistry and Cell Biology, Shanghai Institute for Biological Sciences, Chinese Academy of Sciences (Shanghai, China). Cells were maintained in Dulbecco's Modifed Eagle's Medium (DMEM; Gibco) supplemented with 10\% fetal bovine serum (FBS; Gibco, Gaithersburg, MD) in a $5 \% \mathrm{CO}_{2}, 37^{\circ} \mathrm{C}$ incubator. To establish cisplatin-resistant ovarian cancer cell models, we exposed SKOV3 and A2780 cells to gradually increasing concentrations of cisplatin. Specifically, SKOV3 and A2780 cell lines were initially treated with $1 \mu \mathrm{M}$ cisplatin for 8 weeks. Subsequently, cisplatin concentration was increased every 2 weeks by $0.2 \mu \mathrm{M}$ up to a final concentration of $3 \mu \mathrm{M}$. The established cisplatin-resistant SKOV3 and A2780 cells were named SKOV3-R and A2780-R, respectively. To eliminate the influence of residual cisplatin in the culture medium, SKOV3-R and A2780-R cells were cultured in cisplatin-free DMEM for 2 weeks prior to the experiments.

Quantitative PCR

Total RNA from the ovarian cancer cell lines were extracted by using TRIzol $®$ (Invitrogen, Thermo Fisher Scientific, Inc., Waltham, MA). RNA was reverse-transcribed using the One Step PrimeScript miRNA cDNA Synthesis Kit (TaKaRa, Beijing, China) according to the manufacturer's instructions. Subsequently, miR-139-5p expression was detected using SYBR Premix Ex Taq II (Takara Bio Inc., Kusatsu, Japan) on the ABI PRISM 7900 Sequence Detection System (Applied Biosystems, Foster City, CA). The mature miR-139$5 \mathrm{p}$ amplification primer sequence was 5'-TCTACAGTGCACGTGTCTCCAGT-3'. The relative expression of miR139-5p was determined according to the $2^{-\triangle \Delta \mathrm{Ct}}$ analysis method [18], whereby U6 snRNA was used as the endogenous control. The mature U6 snRNA amplification primer sequence was 5'-CTCGCTTCGGCAGCACA-3'. 


\section{Cellular Physiology Cell Physiol Biochem 2018:51:129-141 and Biochemistry \begin{tabular}{l|l} 
DOI: 10.1159/000495169 & $\begin{array}{l}\text { (c) } 2018 \text { The Author(s). Published by S. Karger AG, Basel } \\
\text { www.karger.com/cpb }\end{array}$
\end{tabular}

Jiang et al.: miR-139 Reverses Cisplatin Resistance in Ovarian Cancer

\section{Transfection}

For knockdown of c-Jun, c-Jun small interfering RNA (siRNA) was purchased from Santa Cruz Biotechnology (Dallas, TX). To construct a c-Jun overexpression vector, the c-Jun open reading frame without the 3' UTR was amplified by PCR with SKOV3 cDNA as a template, and cloned into the HindIII/EcoRI site of the pcDNA3.1 vector (Invitrogen) with the respective restriction enzymes (Takara Bio). The miR-139-5p mimics (5'-UCUACAGUGCACGUGUCUCCAGU-3'), anti-miR-139-5p (5'-ACUGGAGACACGUGCACUGUAGA-3'), and negative control oligonucleotide (NCO, 5'-GCUUACAUUGUCUACCAUGGCGC-3') were purchased from Genepharma Company (Shanghai, China). For transfection, $2 \mu \mathrm{g} / \mathrm{mL} \mathrm{c}$-Jun plasmid, 50 pmol/mL miR-1395p mimics, anti-miR-139-5p, NCO, and c-Jun siRNA were transfected into the ovarian cancer cells by using Lipofectamine 2000 (Invitrogen) according to the manufacturer's instructions. Then, $24 \mathrm{~h}$ after transfection, cells were collected and used for the subsequent experiments.

\section{Cell viability assay}

A total of $5 \times 10^{3}$ transfected ovarian cancer cells were seeded into 96-well plates. After an overnight incubation, cells were treated with different concentrations of cisplatin, oxaliplatin, and carboplatin for 48 h. Cell viability was evaluated by the 3-(4, 5-dimethylthiazol-2-yl)-2, 5-diphenyltetrazolium bromide (MTT) assay [19]. The absorbance in each well was measured at $570 \mathrm{~nm}$ using an enzyme-linked immunoassay microplate reader. The $50 \%$ inhibitory concentration $\left(\mathrm{IC}_{50}\right.$ ) of cisplatin, carboplatin, and oxaliplatin in ovarian cancer cell lines was calculated according to the viability curves.

\section{Luciferase reporter assay}

The c-Jun 3' UTR containing the seed regions of the miR-139-5p binding sites (ACUGUAGA) was cloned into the XhoI/HindIII site of the pGL3 Luciferase Reporter Vector (Promega, Fitchburg, WI) with the respective restriction enzymes (Takara Bio.) according to the manufacturer's instructions. The mutant plasmid pGL3-C-Jun-M was created by mutating the seed regions of the miR-139-5p binding sites (ACUGUAGA) by using a site-directed mutagenesis kit (Takara Bio). To perform the luciferase reporter assay, cells were co-transfected with the pGL3 vector carrying c-Jun 3' UTR, the pRL-TK Renilla luciferase vector control (Promega), and the miR-139-5p mimics (or anti-miR-139-5p) using Lipofectamine 2000. At $48 \mathrm{~h}$ after incubation, the luciferase activity was measured using the Dual-Luciferase Reporter assay system (Promega) according to the manufacturer's instructions. Relative firefly luciferase activity was determined by normalization to Renilla luciferase activity in each well.

\section{Co-immunoprecipitation and western blot analysis}

For co-immunoprecipitation with c-Jun and ATF2, cells were collected and lysed with cold lysis buffer (containing $50 \mathrm{mM}$ Tris [pH 7.4], $150 \mathrm{mM} \mathrm{NaCl}, 1 \%$ NP-40, $1 \mathrm{mM}$ PMSF, $2.5 \mathrm{mM}$ sodium pyrophosphate, $1 \mathrm{mM} \beta$-glycerophosphate, $1 \mathrm{mM} \mathrm{Na}_{3} \mathrm{VO}_{4}$ and $1 \mu \mathrm{g} / \mathrm{mL}$ leupeptin) for $15 \mathrm{~min}$ at $4^{\circ} \mathrm{C}$. Then supernatants were collected after centrifugation at $12,000 \mathrm{~g}$ for $15 \mathrm{~min}$. Subsequently, ATF2 primary antibody (sc-242; Santa Cruz) was added to the protein supernatant and slowly shaken on a rotating shaker at $4^{\circ} \mathrm{C}$ overnight. After incubation with ATF2 antibody, protein A/G plus-Agarose beads (Santa Cruz) were added to the protein lysates for $1 \mathrm{~h}$ at $4^{\circ} \mathrm{C}$. Then immunoprecipitated pellets were mixed with the sodium dodecyl sulfate (SDS) loading buffer and boiled for western blot analysis. For western blot analysis, the extracted proteins were separated by $12.5 \%$ SDS-polyacrylamide gel electrophoresis and transferred to a PVDF membrane (Millipore, Stafford, VA). Then the membranes were probed with primary antibodies for c-Jun (sc-166540), B-cell lymphoma-extra large (Bcl-xl, sc-8392), caspase-9 (sc-133109), caspase-3 (sc-271028), and GAPDH (sc-sc-47724) (all from Santa Cruz) at $4^{\circ} \mathrm{C}$ overnight. Subsequently, the membranes were incubated with appropriate horseradish peroxidase-conjugated secondary antibodies for $2 \mathrm{~h}$ followed by detection with an enhanced chemiluminescence detection kit (Pierce, Thermo Fisher Scientific).

\section{Analysis of mitochondrial membrane potential and apoptosis}

Cells were treated with cisplatin $(15 \mu \mathrm{M})$, miR-139-5p $(50 \mathrm{pmol} / \mathrm{mL})$, and c-Jun plasmid $(2 \mu \mathrm{g} / \mathrm{mL})$ for $24 \mathrm{~h}$. For analysis of the mitochondrial membrane potential, cells were stained with JC-1 (Molecular Probes, Thermo Fisher Scientific) according to the manufacturer's instructions, followed by analysis by flow cytometry (Becton Dickinson, Franklin Lakes, NJ) to detect the red fluorescence, which is indicative of a high mitochondrial membrane potential [20]. For detection of apoptotic rate, cells were stained with Annexin V/ 


\section{Cellular Physiology Cell Physiol Biochem 2018:51:129-141

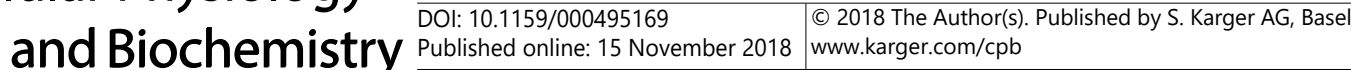 \\ Jiang et al.: miR-139 Reverses Cisplatin Resistance in Ovarian Cancer}

Propidium Iodide (PI) (Sigma-Aldrich, St. Louis, MO) and then analyzed using flow cytometry. The Annexin V-positive and PI-negative cell population was considered early apoptotic cells, the Annexin V-positive and PI-positive cell population was considered late apoptotic cells, and the Annexin V-positive cell population was considered total apoptotic cells.

Tumor growth in nude mice

To evaluate the role of miR-139-5p in vivo, the stable SKOV3-R cell line overexpressing miR-139-5p was generated. Briefly, we purchased the recombinant lentivirus containing the miR-139-5p precursor sequence from Shanghai Genechem Co., Ltd. (Shanghai, China). The precursor sequence of miR-139-5p was 5'-GUGUAUUCUACAGUGCACGUGUCUCCAGUGUGGCUCGGAGGCUGGAGACGCGGCCCUGUUGGAGUAAC-3'. Then $1 \times 10^{4}$ SKOV3-R cells were transfected with $5 \times 10^{5}$ transducing units of lentivirus, and the cells (LTmiR-139-5p) were selected with $1 \mu \mathrm{g} / \mathrm{mL}$ puromycin for 2 weeks. SKOV3-R cells transfected with empty lentiviral vector (LT-control) were used as the control. For inoculation, $5 \times 10^{6}$ prepared SKOV3-R cells were subcutaneously injected into nude mice (BALB/c, nu/nu, 17-22 g; Shanghai Super-B\&K Laboratory Animal Corp., Ltd., Shanghai, China). After xenografts reached $0.5 \mathrm{~cm}$ in diameter, mice were treated with cisplatin intraperitoneal twice a week ( $5 \mathrm{mg} / \mathrm{kg}$ ) until the mice were sacrificed 28 days post-injection. The animal care and experimental protocols were approved by the Animal Care Committee of Cancer Hospital of China Medical University, Liaoning Cancer Hospital \& Institute (Liaoning Sheng, China).

\section{Statistical analysis}

Data are represented as the mean \pm standard deviation (SD), and were obtained from three independent experiments. Statistical analysis was performed using SPSS 16.0 software. For comparison analysis, twotailed Student's $t$-tests were used to estimate the statistical differences between two groups. One-way analysis of variance and Bonferroni's post hoc test were used to determine the differences among three or more groups. P values less than 0.05 were considered statistically significant.

\section{Results}

Expression of miR-139-5p is decreased in cisplatin-resistant ovarian cancer cells

Previous studies have suggested that the expression profile of miR-139-5p is associated with cisplatin sensitivity in ovarian cancer $[16,17]$. Thus, we focused on the potential role of miR-139-5p in the development of cisplatin resistance in ovarian cancer cell lines. To study chemoresistance in ovarian cancer, we established cisplatin-resistant ovarian cancer models of SKOV3 and A2780 cell lines through continuous exposure to cisplatin (SKOV3-R and A2780-R, respectively). As shown in Fig. $1 \mathrm{~A}$, the $\mathrm{IC}_{50}$ of cisplatin against SKOV3-R and A2780-R was significantly higher than that in their parental cell lines SKOV3 and A2780, respectively. However, the results of the qPCR analysis showed that miR-139-5p expression in SKOV3-R and A2780-R cells was decreased compared to SKOV3 and A2780 cells, respectively (Fig. 1B). These results suggest that the downregulation of miR-139-5p is associated with cisplatin resistance in ovarian cancer.

Recovery of miR-139-5p reverses cisplatin resistance in ovarian cancer

To investigate the role of miR-139-5p in the development of cisplatin resistance in ovarian cancer, we restored miR-139-5p expression in SKOV3-R and A2780-R cells by transfecting them with miR-139-5p mimics. Then we knocked down miR-139-5p expression in routine SKOV3 and A2780 cells using miR-139-5p antisense-oligonucleotides (anti-miR139-5p). The transfection efficiency of miR-139-5p and anti-miR-139-5p is shown in Fig. $2 \mathrm{~A}$. The results of the cell viability assays showed that recovery of miR-139-5p significantly increased the sensitivity of SKOV3-R and A2780-R cells to cisplatin treatment (Fig. 2B). Meanwhile, knockdown of miR-139-5p in parental SKOV3 and A2780 cells induced clear resistance to cisplatin treatment (Fig. 2C). These results suggest that the expression profile of miR-139-5p affects chemosensitivity in ovarian cancer. Furthermore, the combination of cisplatin with miR-139-5p may represent a potential strategy for reversing cisplatin resistance in ovarian cancer. 


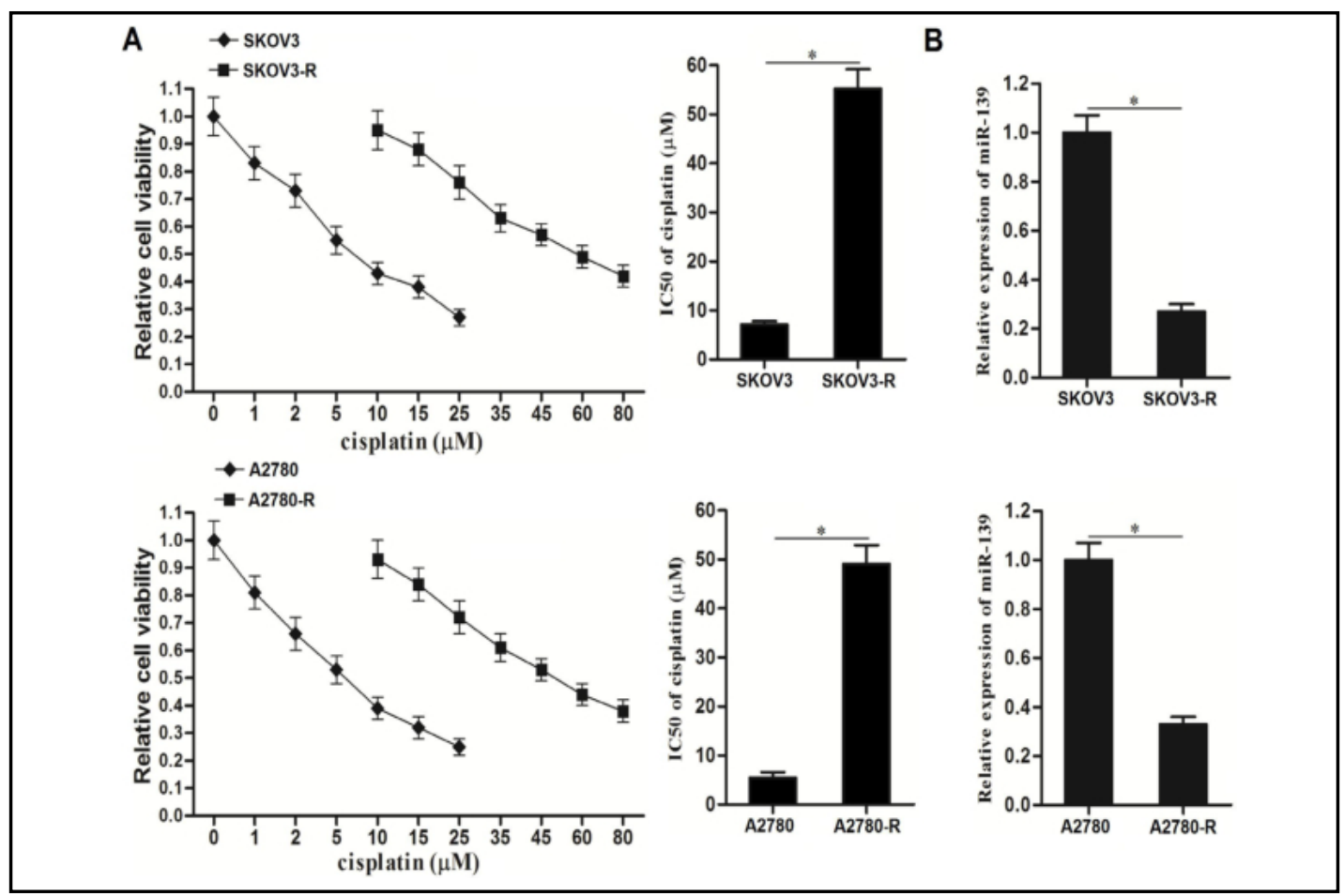

Fig. 1. Dysregulation of miR-139-5p in cisplatin-resistant ovarian cancer cells. A: After treatment with different concentrations of cisplatin for 48 h, cell viability of SKOV3, SKOV3-R, A2780 and A2780-R was measured using MTT assays. Cisplatin $\mathrm{IC}_{50}$ was determined according to the cell viability curve. ${ }^{*} \mathrm{P}<0.05$. $\mathrm{B}$ : qPCR analysis was performed to detect the relative expression of miR-139-5p in SKOV3, SKOV3-R, A2780, and A2780-R cells ( ${ }^{*} \mathrm{P}<0.05$ ).

Fig. 2. Role of miR-139-5p in cisplatin resistance in ovarian cancer. A: Transfection efficiency of miR139-5p mimics and antisenseoligonucleotides (50 $\mathrm{pmol} / \mathrm{mL}$ ) on SKOV3-R, A2780-R, SKOV3, and A2780 cells $\left({ }^{*} \mathrm{P}<0.05\right) . \quad \mathrm{B}$ : Effects of miR-139$5 \mathrm{p}$ on decreasing the $\mathrm{IC}_{50}$ of cisplatin in SKOV3-R and A2780-R cells ( $\left.{ }^{*} \mathrm{P}<0.05\right)$. C: Effects

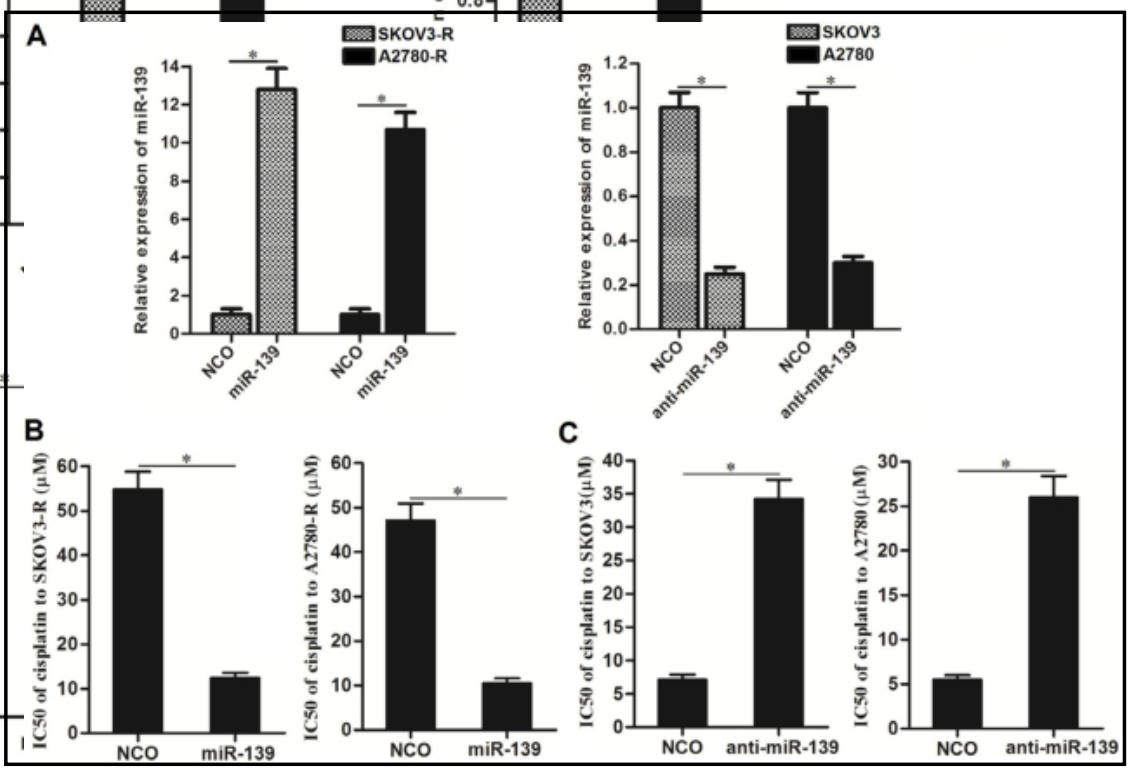
of anti-miR-139-5p on increasing the $\mathrm{IC}_{50}$ of cisplatin in SKOV3 and A2780 cells $\left({ }^{*} \mathrm{P}<0.05\right)$. 


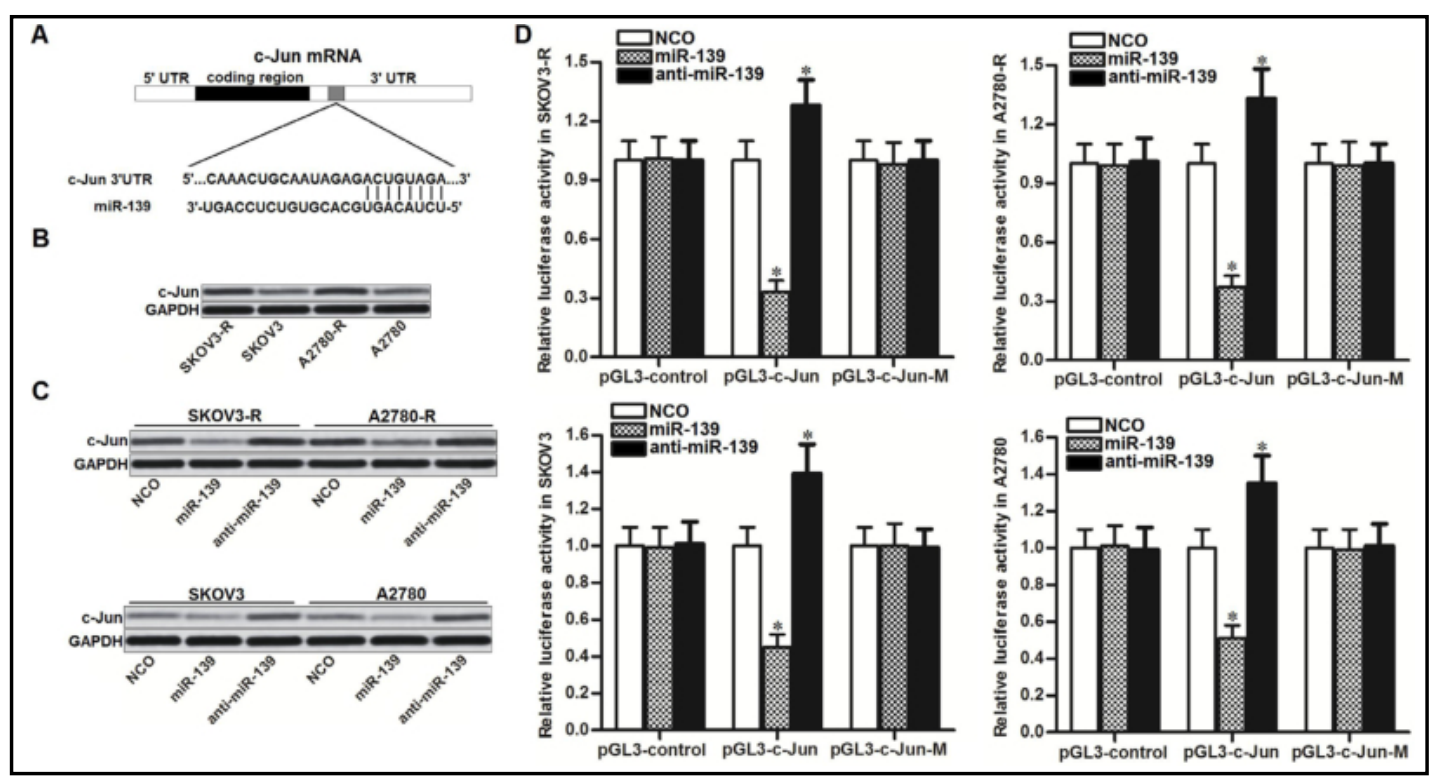

Fig. 3. c-Jun is the target of miR-139-5p in ovarian cancer. A: Sequence of c-Jun $3^{\prime}$ UTR seed region paired with miR-139-5p. B: Western blot analysis was performed to evaluate the expression of c-Jun in SKOV3-R, SKOV3, A2780-R, and A2780 cells. C: Protein level of c-Jun in ovarian cancer cell lines after transfection with miR-139-5p or anti-miR-139-5p. D: Luciferase activity in SKOV3-R, SKOV3, A2780-R, and A2780 cells, as measured by the dual-luciferase reporter assay system ( ${ }^{*} \mathrm{P}<0.05$ vs. the NCO group).

\section{miR-139-5p targets c-Jun in ovarian cancer}

To understand how miR-139-5p reversed cisplatin resistance in ovarian cancer, TargetScan, miRanda, and PicTar databases were used to search for potential mRNA targets. All of these databases showed that the proto-oncogene c-Jun contained a putative binding sequence paired with miR-139-5p at the $3^{\prime}$ UTR of its mRNA (Fig. 3A). Furthermore, in contrast to the downregulation of miR-139-5p that was observed in cisplatin-resistant ovarian cancer cells, the expression level of c-Jun was increased in SKOV3-R and A2780-R cells compared to their parental SKOV3 and A2780 cells (Fig. 3B). Therefore, we predicted that miR-139-5p targets c-Jun in ovarian cancer. To confirm the miR-139-5p/c-Jun axis in ovarian cancer, we evaluated c-Jun protein in ovarian cancer cell lines after they were transfected with miR-139-5p or anti-miR-139-5p. The overexpression of miR-139-5p clearly decreased the protein level of c-Jun, whereas knockdown of miR-139-5p decreased the expression of c-Jun in SKOV3-R, A2780-R, and their parental SKOV3 and A2780 cells (Fig. 3C). The results of the luciferase reporter assays showed that co-transfection with miR-139$5 p$ significantly decreased the luciferase activity of pGL3-c-Jun reporters, whereas anti-miR139-5p obviously increased the luciferase activity of pGL3-c-Jun reporters. However, both miR-139-5p and anti-miR-139-5p failed to regulate the luciferase activity of pGL3 reporters carrying the mutant c-Jun 3' UTR at the predicted miR-139-5p binding sites (Fig. 3D). Taken together, these data demonstrate that miR-139-5p targets c-Jun in ovarian cancer.

Recovery of miR-139-5p reverses cisplatin resistance in ovarian cancer through the miR139-5p/c-Jun axis

Because c-Jun was proven to be regulated by miR-139-5p in ovarian cancer, we next studied the role of the miR-139-5p/c-Jun axis in regulating cisplatin resistance in ovarian cancer. To perform c-Jun gain-of-function and loss-of-function experiments, we transfected SKOV3-R and A2780-R cells with a c-Jun eukaryotic expression plasmid and c-Jun siRNA, respectively. As shown in Fig. 4A, transfection with c-Jun siRNA directly knocked down c-Jun, while transfection with c-Jun plasmid abolished the effects of miR-139-5p on decreasing 


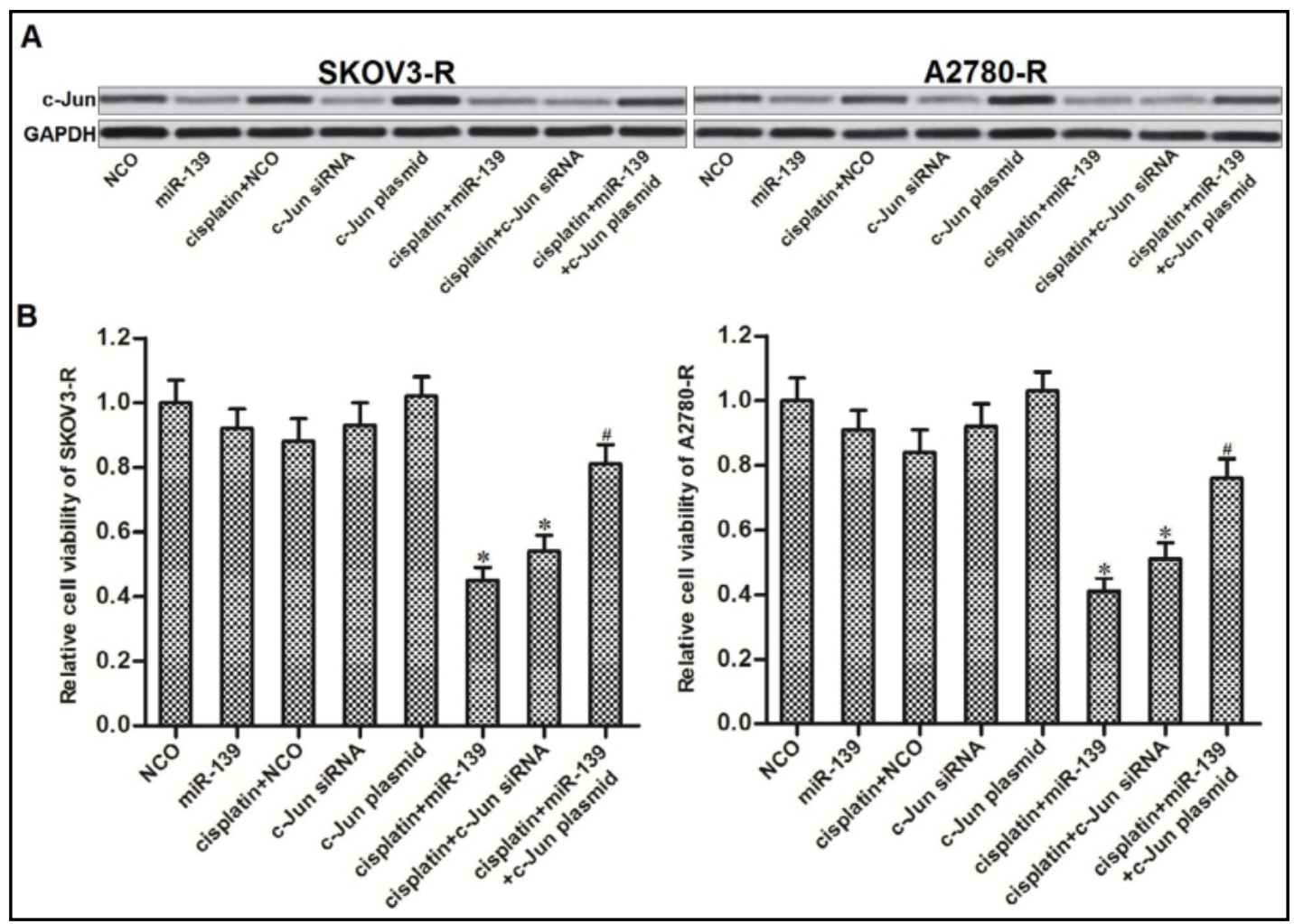

Fig. 4. Recovery of miR-139-5p reverses cisplatin resistance in ovarian cancer through the miR-139-5p/cJun axis. A: Western blot analysis to evaluate transfection efficiency of c-Jun siRNA and c-Jun plasmid in SKOV3-R and A2780-R cells. B: MTT assays to test the effects of c-Jun siRNA and c-Jun plasmid on regulating viability of SKOV3-R and A2780-R cells treated with miR-139-5p and cisplatin $\left(15 \mu \mathrm{M} ;{ }^{*} \mathrm{P}<0.05\right.$ vs. cisplatin + NCO group, ${ }^{\#} \mathrm{P}<0.05$ vs. cisplatin + miR-139-5p group).

c-Jun expression. The results of the MTT assays showed that transfection with c-Jun siRNA clearly sensitized SKOV3-R and A2780-R cells to cisplatin treatment, similar to the effects observed with miR-139-5p. However, overexpression of c-Jun inhibited miR-139-5p-induced cell death in cisplatin-treated SKOV3-R and A2780-R cells (Fig. 4B). Taken together, these results suggest that recovery of miR-139-5p reverses cisplatin resistance in ovarian cancer through the miR-139-5p/c-Jun axis.

Recovery of miR-139-5p enhances cisplatin-induced apoptosis in cisplatin-resistant ovarian cancer through the c-Jun/Bcl-xl pathway

ATF2 is a common co-activator with c-Jun. Formation of c-Jun/ATF2 heterodimers induces the overexpression of Bcl-xl in cancer cells [21, 22]. We observed that cisplatin treatment in SKOV3-R and A2780-R cells induced an obvious interaction with c-Jun and ATF2. However, co-treatment of cisplatin with miR-139-5p suppressed the expression of c-Jun, thereby inhibiting the cisplatin-induced interaction of ATF2 with c-Jun (Fig. 5A). Furthermore, despite cisplatin treatment-induced overexpression of $\mathrm{Bcl}-\mathrm{xl}$, the combination of cisplatin with miR-139-5p clearly decreased the expression of Bcl-xl in SKOV3-R and A2780-R cells. However, the overexpression of c-Jun abolished the effects of miR-139-5p on suppressing Bcl-xl expression (Fig. 5B). These data suggest that the miR-139-5p/c-Jun axis regulates the expression of $\mathrm{Bcl}-\mathrm{xl}$ in cisplatin-resistant ovarian cancer. As Bcl-xl is a key prosurvival protein that suppresses mitochondrial apoptosis, we studied the role of miR-139-5p in the cisplatin-induced mitochondrial apoptosis pathway. As shown in Fig. 5C, recovery of miR-139-5p enhanced the effects of cisplatin in inducing mitochondrial membrane collapse in SKOV3-R and A2780-R cells. However, overexpression of c-Jun protected the mitochondria 


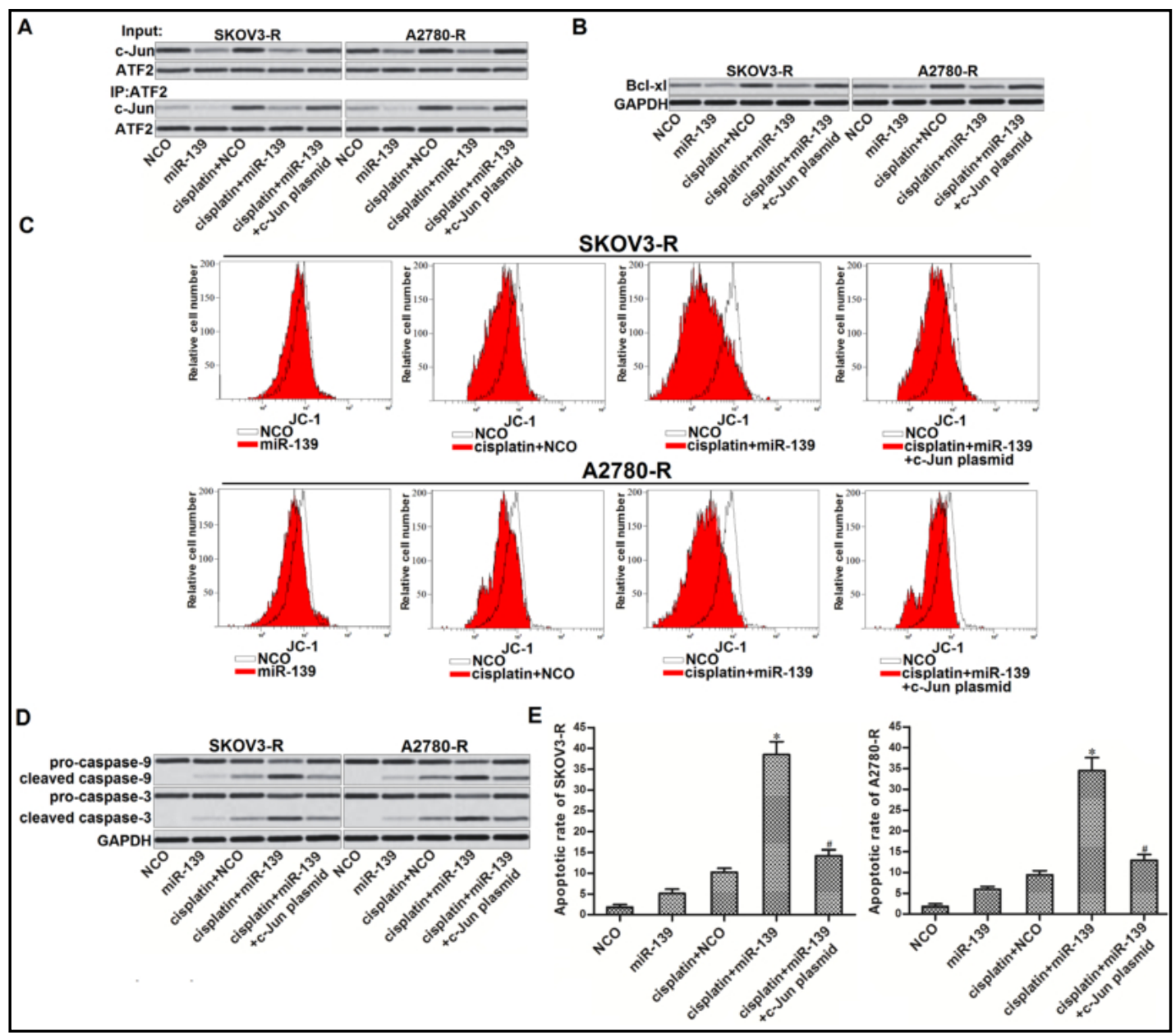

Fig. 5. miR-139-5p targets the c-Jun/Bcl-xl pathway to enhance cisplatin-induced apoptosis in cisplatinresistant ovarian cancer through the c-Jun/Bcl-xl pathway. A: Co-immunoprecipitation and western blot analysis to evaluate the interaction with ATF2 and c-Jun in SKOV3-R and A2780-R cells. B: Western blot analysis to evaluate Bcl-xl expression level in SKOV3-R and A2780-R cells. C: Flow cytometry analysis to detect mitochondrial membrane potential of SKOV3-R and A2780-R cells. D: Cleavage of caspase-9 and caspase-3 in SKOV3-R and A2780-R cells treated with cisplatin $(15 \mu \mathrm{M})$, miR-139-5p (50 pmol/mL), and c-Jun plasmid $(2 \mu \mathrm{g} / \mathrm{mL})$. E: Flow cytometry to detect the apoptotic rate of SKOV3-R and A2780-R cells treated with cisplatin $(15 \mu \mathrm{M})$, miR-139-5p (50 pmol/mL), and c-Jun plasmid $(2 \mu \mathrm{g} / \mathrm{mL})$.

from the damage of miR-139-5p and cisplatin co-treatment. Western blotanalysis showed that the combination of cisplatin and miR-139-5p induced cleavage of caspase- 9 and caspase-3, whereas transfection with c-Jun plasmid weakened the activation of these caspases (Fig. 5D). Accompanied by caspase cleavage, combination treatment of cisplatin and miR-139-5p induced a higher level of apoptosis compared to treatment with cisplatin alone (Fig. 5E). These data demonstrate that the miR-139-5p/c-Jun axis suppresses the expression of Bcl-xl, thereby promoting cisplatin-induced apoptosis in cisplatin-resistant ovarian cancer.

Overexpression of miR-139-5p sensitizes cisplatin-resistant ovarian cancer cells to cisplatin in vivo

To investigate the role of miR-139-5p in reversing cisplatin resistance in ovarian cancer in vivo, we established an in vivo model of ovarian cancer by using SKOV3-R cells. With an equal dose of cisplatin treatment, the growth rate of tumors overexpressing miR-139-5p was slower than that in LT-control tumors (Fig. 6A), indicating that miR-139-5p can enhance the anti-tumor effects of cisplatin in ovarian cancer. After euthanasia in mice, tumor tissues 


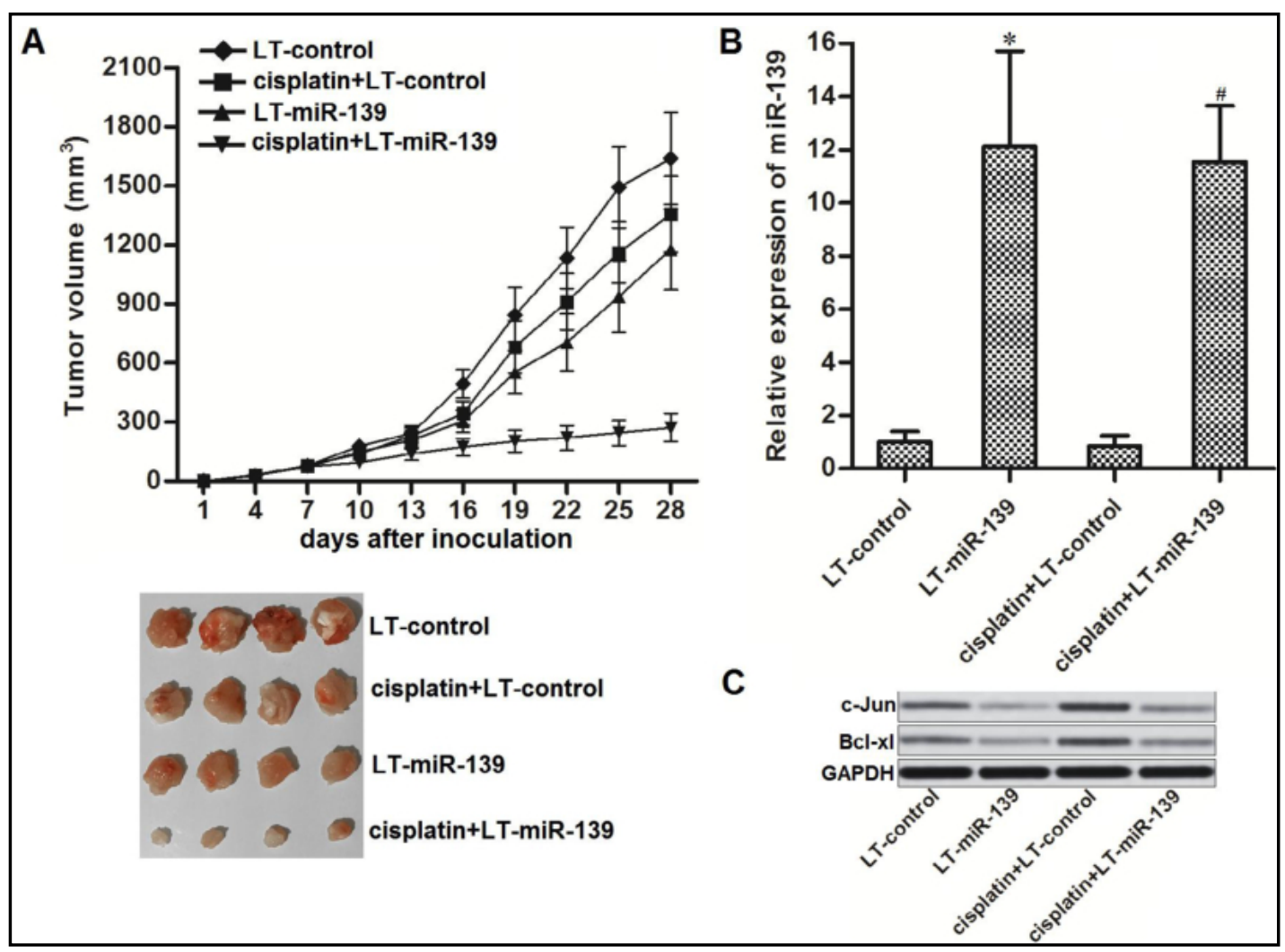

Fig. 6. miR-139-5p sensitizes cisplatin-resistant ovarian cancer cells to cisplatin in vivo. A: Nude mice were inoculated with LT-miR-139-5p or LT-control SKOV3-R cells before treatment with cisplatin (5 mg/kg) twice a week. Tumor volumes were detected every 3 days until the experimental end-point (28 days postinjection). B: Expression of miR-139-5p in separated tumor tissues was measured by qPCR analysis $\left({ }^{*} \mathrm{P}<0.05\right.$ vs. LT-control group, " $\mathrm{P}<0.05$ vs. cisplatin + LT-control group). C: Western blot analysis was performed to evaluate the expression of c-Jun and Bcl-xl in tumor tissues.

were separated. We observed that miR-139-5p expression was increased in LT-miR-1395p-transfected tumor tissues compared to the LT-control tumors (Fig. 6B). In contrast, the LT-miR-139-5p tumors expressed markedly lower levels of c-Jun and Bcl-xl with cisplatin treatment (Fig. 6C). Taken together, these data demonstrate that overexpression of miR-139$5 p$ sensitizes cisplatin-resistant ovarian cancer cells to cisplatin in vivo through suppression of c-Jun.

Recovery of miR-139-5p decreases the cross resistance of ovarian cancer cells to other platinum-based chemotherapeutic drugs

The results of the cell viability assays showed that the cisplatin-resistant ovarian cancer cell lines SKOV3-R and A2780-R exhibited cross-resistance to carboplatin (Fig. 7A) and oxaliplatin (Fig. 7B). We investigated the effects of miR-139-5p on oxaliplatin- and carboplatin-induced cytotoxicity against SKOV3-R and A2780-R cells. We found that recovery of miR-139-5p expression decreased the $\mathrm{IC}_{50}$ of SKOV3-R and A2780-R cells to carboplatin (Fig. 7C) and oxaliplatin (Fig. 7D). Thus, overexpression of miR-139-5p decreases the crossresistance of ovarian cancer cells to platinum-based chemotherapy. 


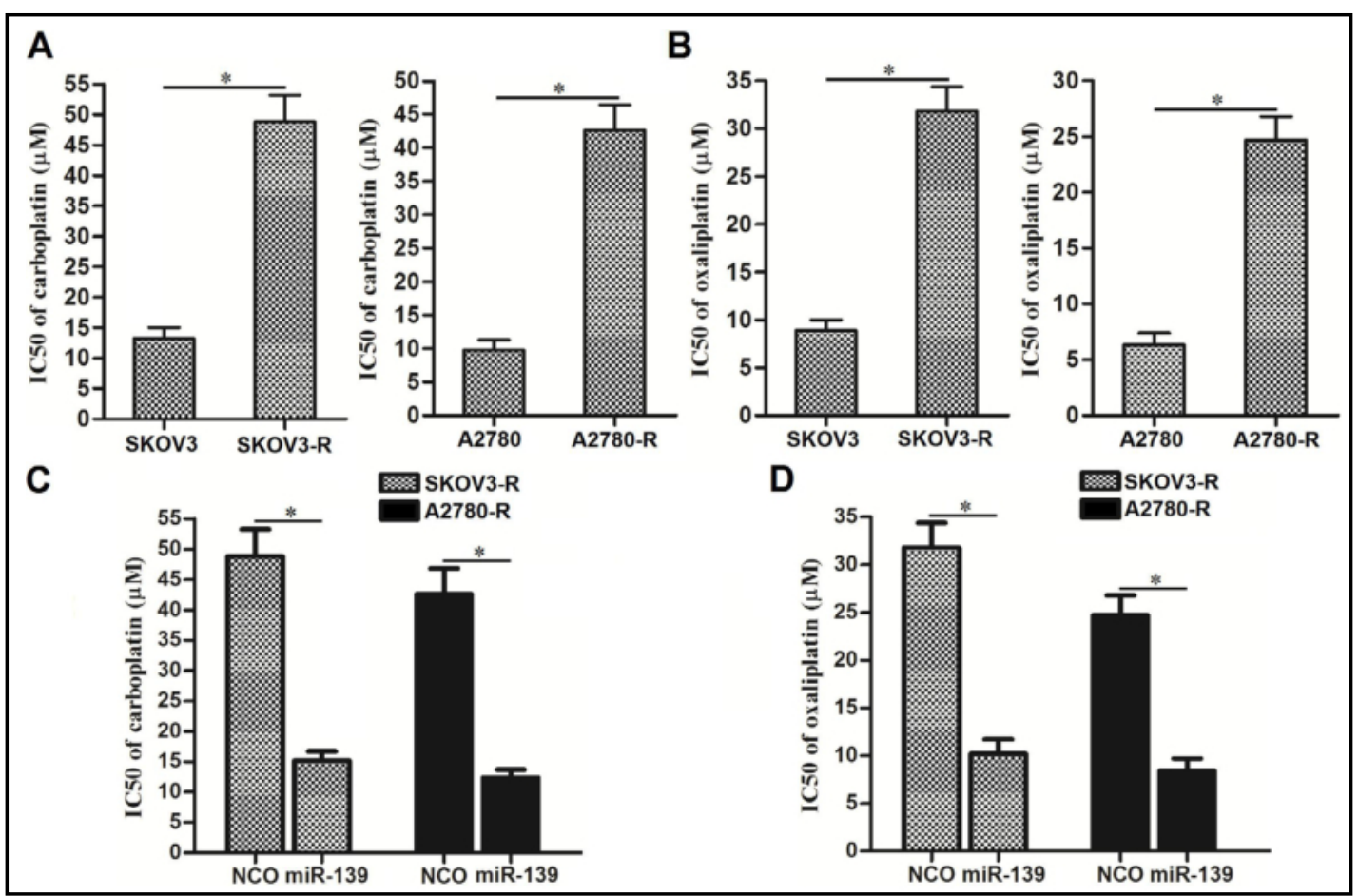

Fig. 7. Effects of miR-139-5p on reversing cross-resistance of ovarian cancer cells to platinum-based chemotherapeutic drugs. A: Carboplatin $\mathrm{IC}_{50}$ SKOV3, SKOV3-R, A2780, and A2780-R cells $\left({ }^{*} \mathrm{P}<0.05\right)$. B: Oxaliplatin $\mathrm{IC}_{50}$ to SKOV3, SKOV3-R, A2780, and A2780-R cells ( $\left.{ }^{*} \mathrm{P}<0.05\right)$. C: Effects of miR-139-5p (50 pmol/ $\mathrm{mL}$ ) on decreasing carboplatin $\mathrm{IC}_{50}$ to SKOV3-R and A2780-R cell lines ( $\left.{ }^{*} \mathrm{P}<0.05\right)$. D: Effects of miR-139-5p $(50 \mathrm{pmol} / \mathrm{mL})$ on decreasing oxaliplatin $\mathrm{IC}_{50}$ to SKOV3-R and A2780-R cell lines $\left({ }^{*} \mathrm{P}<0.05\right)$.

\section{Discussion}

Cisplatin, a platinum-based chemotherapeutic drug, is effectively and widely used for the treatment of multiple cancers including ovarian cancer [23-25]. Although cisplatin initially induces significant apoptosis in cancer cells, cancer cells can develop mechanisms to escape the apoptotic pathway after a period of cisplatin administration [26-29]. Thus, there is a need to develop effective strategies for preventing or reversing cisplatin resistance.

MiRNAs regulate nearly every cellular process in cancer cells, and their dysregulation has been shown to induce drug resistance and a low response to chemotherapy [30, 31]. Among these cancer-related miRNAs, miR-139-5p is usually downregulated in cancers and has been shown to suppress cell proliferation, migration and invasion in several cancers such as glioblastoma, colorectal cancer, non-small cell lung cancer, and ovarian cancer [3235]. More importantly, the downregulation of miR-139-5p is reportedly associated with multidrug resistance in colorectal and breast cancers [36-38]. However, the role of miR$139-5 p$ in the chemoresistance of ovarian cancer is unclear.

In this study, we established cisplatin-resistant ovarian cancer cell models. Our findings showed that the expression profile of miR-139-5p was significantly downregulated in cisplatin-resistant ovarian cancer cells compared to their parental cells. We therefore demonstrated that miR-139-5p determines cisplatin sensitivity in ovarian cancer. Next, we increased the cellular level of miR-139-5p in cisplatin-resistant ovarian cancer cells by transfection with miR-139-5p mimics. The recovery of miR-139-5p reversed the cisplatin resistance of ovarian cancer both in vitro and in vivo. Thus, our work showed that miR-139$5 p$ is a potential therapeutic target for improving cisplatin-based chemotherapy in ovarian cancer. 


\section{Cellular Physiology Cell Physiol Biochem 2018;51:129-141 \begin{tabular}{l|l|l|l|l} 
DOl: 10.1159/000495169 & O 2018 The Author(s). Published by S. Karger AG, Basel \\
and Biochemistry
\end{tabular}

c-Jun is an important component of the activation protein-1 (AP-1) transcription factor. In cancer cells, c-Jun functions as a proto-oncogene that promotes cell proliferation, invasion, and metastasis $[39,40]$. Moreover, overexpression of c-Jun can repair DNA damage, thus preventing the incidence of apoptosis in cancer cells $[41,42]$. It has been reported that c-Jun overexpression is responsible for cisplatin resistance in cancers including ovarian cancer [43-46]. However, the mechanisms of c-Jun overexpression in cisplatin resistance remain unknown. A previous study suggested a correlation between miR-139 and c-Jun expression in gastric cancer [47]. However, the role of miR-139 in regulating c-Jun expression is remains to be explored. In this study, we demonstrated that decreasing miR-139-5p expression induced the overexpression of c-Jun in cisplatin-resistant ovarian cancer. We then proved that cisplatin resistance in ovarian cancer was associated with the miR-139-5p/c-Jun axis. Moreover, our findings showed that recovery of miR-139-5p reversed cisplatin resistance in ovarian cancer through the inhibition of c-Jun expression.

ATF2 belongs to the cAMP response element binding family [48], and overexpressed c-Jun can interact with ATF2 to promote the expression of Bcl-xl. As Bcl-xl is a key antiapoptotic protein, the c-Jun/ATF2 complex acts as a negative regulator of mitochondrial apoptosis [49-51]. In this study, we found that recovery of miR-139-5p in cisplatin-resistant ovarian cancer cells inhibited the interaction with c-Jun and ATF2, and thus decreased the expression of Bcl-xl. As a result, we found that the sensitivity of cisplatin-resistant ovarian cancer cells to cisplatin-induced apoptosis could be significantly increased due to the recovery of miR-139-5p.

\section{Conclusion}

Taken together, our findings provide several pieces of evidence demonstrating the effects of miR-139-5p in reversing the resistance of ovarian cancer cells to platinum-based chemotherapy. However, further studies are required to evaluate the novel strategy of targeting miR-139-5p as an adjuvant treatment strategy for clinical applications.

\section{Acknowledgements}

This work was supported by the Natural Science Foundation of Liaoning Province, China (Grant No. 20170540570).

\section{Disclosure Statement}

The authors have no conflicts of interest to declare.

\section{References}

$>1$ Siegel RL, Miller KD, Jemal A: Cancer statistics, 2015. CA Cancer J Clin 2015;65:5-29.

-2 Liu X, Ma L, Rao Q Mao Y, Xin Y, Xu H, Li C, Wang X: MiR-1271 Inhibits Ovarian Cancer Growth by Targeting Cyclin G1. Med Sci Monit 2015;21:3152-3158.

- Vergote I, Amant F, Kristensen G, Ehlen T, Reed NS, Casado A: Primary surgery or neoadjuvant chemotherapy followed by interval debulking surgery in advanced ovarian cancer. Eur J Cancer 2011;47 Suppl 3:S88-92.

4 Xu S, Fu GB, Tao Z, OuYang J, Kong F, Jiang BH, Wan X, Chen K: MiR-497 decreases cisplatin resistance in ovarian cancer cells by targeting mTOR/P70S6K1. Oncotarget 2015;6:26457-26471.

5 Morgan SL, Medina JE, Taylor MM, Dinulescu DM: Targeting platinum resistant disease in ovarian cancer. Curr Med Chem 2014;21:3009-3020. 


\section{Cellular Physiology Cell Physiol Biochem 2018;51:129-141 and Biochemistry \begin{tabular}{l|l} 
DOI: $10.1159 / 000495169$ & (c) 2018 The Author(s). Published by S. Karger AG, Basel \\
www.karger.com/cpb
\end{tabular}

6 Xie Q, Wang S, Zhao Y, Zhang Z, Qin C, Yang X: MiR-519d impedes cisplatin-resistance in breast cancer stem cells by down-regulating the expression of MCL-1. Oncotarget 2017;8:22003-22013.

-7 Xu YY, Wu TT, Zhou SH, Bao YY, Wang QY, Fan J, Huang YP: Apigenin suppresses GLUT-1 and p-AKT expression to enhance the chemosensitivity to cisplatin of laryngeal carcinoma Hep-2 cells: an in vitro study. Int J Clin Exp Pathol 2014;7:3938-3947.

-8 Yu PN, Yan MD, Lai HC, Huang RL, Chou YC, Lin WC, Yeh LT, Lin YW: Downregulation of miR-29 contributes to cisplatin resistance of ovarian cancer cells. Int J Cancer 2014;134:542-551.

-9 Wu H, Wang K, Liu W, Hao Q: SPTEN overexpression improves cisplatin-resistance of human ovarian cancer cells through upregulating KRT10 expression. Biochem Biophys Res Commun 2014;444:141-146.

10 Bartel DP: MicroRNAs: target recognition and regulatory functions. Cell 2009;136:215-233.

11 Iorio MV and Croce CM: microRNA involvement in human cancer. Carcinogenesis 2012;33:1126-1133.

12 Di Leva G, Garofalo M, Croce CM: MicroRNAs in cancer. Annu Rev Pathol 2014; 9:287-314.

13 Pan C, Wang X, Shi K, Zheng Y, Li J, Chen Y, Jin L, Pan Z: MiR-122 Reverses the Doxorubicin-Resistance in Hepatocellular Carcinoma Cells through Regulating the Tumor Metabolism. PLoS One 2016;11:e0152090.

14 Jiang J, Feng X, Zhou W, Wu Y, Yang Y: MiR-128 reverses the gefitinib resistance of the lung cancer stem cells by inhibiting the c-met/PI3K/AKT pathway. Oncotarget 2016;7:73188-73199.

15 Liu W, Wang S, Zhou S, Yang F, Jiang W, Zhang Q, Wang L: A systems biology approach to identify microRNAs contributing to cisplatin resistance in human ovarian cancer cells. Mol Biosyst 2017;13:2268-2276.

16 Xiao F, Li Y, Wan Y, Xue M: MircroRNA-139 sensitizes ovarian cancer cell to cisplatin-based chemotherapy through regulation of ATP7A/B. Cancer Chemother Pharmacol 2018;81:935-947.

17 Chen Y, Cao XY, Li YN, Qiu YY, Li YN, Li W, Wang H: Reversal of cisplatin resistance by microRNA-139-5pindependent RNF2 downregulation and MAPK inhibition in ovarian cancer. Am J Physiol Cell Physiol 2018.

-18 Livak KJ, Schmittgen TD: Analysis of relative gene expression data using real-time quantitative PCR and the 2(-Delta Delta C(T)) method. Methods 2001;25:402-408.

19 Wang J, Tian X, Han R, Zhang X, Wang X, Shen H, Xue L, Liu Y, Yan X, Shen J, et al: Downregulation of miR486-5p contributes to tumor progression and metastasis by targeting protumorigenic ARHGAP5 in lung cancer. Oncogene 2014;33:1181-1189.

20 Jiang K, Wang W, Jin X, Wang Z, Ji Z, Meng G: Silibinin, a natural flavonoid, induces autophagy via ROSdependent mitochondrial dysfunction and loss of ATP involving BNIP3 in human MCF7 breast cancer cells. Oncol Rep 2015;33:2711-2718.

21 Chen YJ, Chang LS: Simvastatin induces NFKB/p65 down-regulation and JNK1/c-Jun/ATF-2 activation, leading to matrix metalloproteinase-9 (MMP-9) but not MMP-2 down-regulation in human leukemia cells. Biochem Pharmacol 2014;92:530-543.

22 Salameh A, Galvagni F, Anselmi F, De Clemente C, Orlandini M, Oliviero S: Growth factor stimulation induces cell survival by c-Jun. ATF2-dependent activation of Bcl-XL. J Biol Chem 2010;285:23096-23104.

23 Yu PN, Yan MD, Lai HC, Huang RL, Chou YC, Lin WC, Yeh LT, Lin YW: Downregulation of miR-29 contributes to cisplatin resistance of ovarian cancer cells. Int J Cancer 2014;134:542-551.

-24 Chen H, Xin Y, Zhou L, Huang JM, Tao L, Cheng L, Tian J: Cisplatin and paclitaxel target significant long noncoding RNAs in laryngeal squamous cell carcinoma. Med Oncol 2014;31:246.

-25 Takekuma M, Kuji S, Tanaka A, Takahashi N, Abe M, Hirashima Y: Platinum sensitivity and non-crossresistance of cisplatin analogue with cisplatin in recurrent cervical cancer. J Gynecol Oncol 2015;26:185192.

-26 Galluzzi L, Senovilla L, Vitale I, Michels J, Martins I, Kepp 0, Castedo M, Kroemer G: Molecular mechanisms of cisplatin resistance. Oncogene 2012;31:1869-1883.

27 Yang X, Yang Y, Tang S, Tang H, Yang G, Xu Q Wu J: EphB4 inhibitor overcome the acquired resistance to cisplatin in melanomas xenograft model. J Pharmacol Sci 2015;129:65-71.

28 Wen K, Fu Z, Wu X, Feng J, Chen W, Qian J: Oct-4 is required for an antiapoptotic behavior of chemoresistant colorectal cancer cells enriched for cancer stem cells: effects associated with STAT3/Survivin. Cancer Lett 2013;333:56-65.

29 Van Houdt WJ, Emmink BL, Pham TV, Piersma SR, Verheem A, Vries RG, et al: Comparative proteomics of colon cancer stem cells and differentiated tumor cells identifies BIRC6 as a potential therapeutic target. Mol Cell Proteomics 2011;10:M111.011353.

-30 Chen Y, Gao Y, Zhang K, Li C, Pan Y, Chen J, Wang R, Chen L: MicroRNAs as Regulators of Cisplatin Resistance in Lung Cancer. Cell Physiol Biochem 2015;37:1869-1880. 


\section{Cellular Physiology Cell Physiol Biochem 2018;51:129-141 and Biochemistry \begin{tabular}{l|l} 
DOI: $10.1159 / 000495169$ & (c) 2018 The Author(s). Published by S. Karger AG, Basel \\
www.karger.com/cpb
\end{tabular}

Jiang et al.: miR-139 Reverses Cisplatin Resistance in Ovarian Cancer

-31 Pouliot LM, Shen DW, Suzuki T, Hall MD, Gottesman MM: Contributions of microRNA dysregulation to cisplatin resistance in adenocarcinoma cells. Exp Cell Res 2013;319:566-574.

-32 Song M, Yin Y, Zhang J, Zhang B, Bian Z, Quan C, Zhou L, Hu Y, Wang Q Ni S, Fei B, Wang W, Du X, Hua D, Huang Z: MiR-139-5p inhibits migration and invasion of colorectal cancer by downregulating AMFR and NOTCH1. Protein Cell 2014;5:851-861.

-33 Yue S, Wang L, Zhang H, Min Y, Lou Y, Sun H, Jiang Y, Zhang W, Liang A, Guo Y, Chen P, Lv G, Wang L, Zong Q Li Y: miR-139-5p suppresses cancer cell migration and invasion through targeting ZEB1 and ZEB2 in GBM. Tumour Biol 2015;36:6741-6749.

34 Sun C, Sang M, Li S, Sun X, Yang C, Xi Y, Wang L, Zhang F, Bi Y, Fu Y, Li D: Hsa-miR-139-5p inhibits proliferation and causes apoptosis associated with down-regulation of c-Met. Oncotarget 2015;6:3975639792.

35 Liu J, Jin S, Wang R: MicroRNA-139 suppressed tumor cell proliferation, migration and invasion by directly targeting HDGF in epithelial ovarian cancer. Mol Med Rep 2017;16:3379-3386.

-36 Xu K, Shen K, Liang X, Li Y, Nagao N, Li J, Liu J, Yin P: MiR-139-5p reverses CD44+/CD133+-associated multidrug resistance by downregulating NOTCH1 in colorectal carcinoma cells. Oncotarget 2016;7:7511875129.

37 Liu H, Yin Y, Hu Y, Feng Y, Bian Z, Yao S, Li M, You Q, Huang Z: miR-139-5p sensitizes colorectal cancer cells to 5-fluorouracil by targeting NOTCH-1. Pathol Res Pract 2016;212:643-649.

-38 Zhang HD, Sun DW, Mao L, Zhang J, Jiang LH, Li J, Wu Y, Ji H, Chen W, Wang J, Ma R, Cao HX, Wu JZ, Tang JH: MiR-139-5p inhibits the biological function of breast cancer cells by targeting Notch1 and mediates chemosensitivity to docetaxel. Biochem Biophys Res Commun 2015;465:702-713.

39 Peng Y, Zhang P, Huang X, Yan Q, Wu M, Xie R, Wu Y, Zhang M, Nan Q, Zhao J, Li A, Xiong J, Ren Y, Bai Y, Chen Y, Liu S, Wang J: Direct regulation of FOXK1 by C-jun promotes proliferation, invasion and metastasis in gastric cancer cells. Cell Death Dis 2016;7:e2480.

40 Zhang Y, Pu X, Shi M, Chen L, Song Y, Qian L, Yuan G, Zhang H, Yu M, Hu M, Shen B, Guo N: Critical role of c-Jun overexpression in liver metastasis of human breast cancer xenograft model. BMC Cancer 2007;7:145.

-41 Salomoni P, Bernardi R, Bergmann S, Changou A, Tuttle S, Pandolfi PP: The promyelocytic leukemia protein PML regulates c-Jun function in response to DNA damage. Blood 2005;105:3686-3690.

-42 Potapova O, Basu S, Mercola D, Holbrook NJ: Protective role for c-Jun in the cellular response to DNA damage. J Biol Chem 2001;276:28546-28553.

43 Xia Y, Yang W, Bu W, Ji H, Zhao X, Zheng Y, Lin X, Li Y, Lu Z: Differential regulation of c-Jun protein plays an instrumental role in chemoresistance of cancer cells. J Biol Chem 2013;288:19321-19329.

44 Zhao R, Rabo YB, Egyházi S, Andersson A, Edgren MR, Linder S, Hansson J. Apoptosis and c-jun induction by cisplatin in a human melanoma cell line and a drug-resistant daughter cell line. Anticancer Drugs 1995;6:657-668.

45 Pan B, Yao KS, Monia BP, Dean NM, McKay RA, Hamilton TC, O’Dwyer PJ: Reversal of cisplatin resistance in human ovarian cancer cell lines by a c-jun antisense oligodeoxynucleotide (ISIS 10582): evidence for the role of transcription factor overexpression in determining resistant phenotype. Biochem Pharmacol 2002;63:1699-1707.

-46 Mansouri A, Ridgway LD, Korapati AL, Zhang Q, Tian L, Wang Y, Siddik ZH, Mills GB, Claret FX: Sustained activation of JNK/p38 MAPK pathways in response to cisplatin leads to Fas ligand induction and cell death in ovarian carcinoma cells. J Biol Chem 2003;278:19245-19256.

47 Zhang Y, Shen WL, Shi ML, Zhang LZ, Zhang Z, Li P, Xing LY, Luo FY, Sun Q Zheng XF, Yang X, Zhao ZH: Involvement of aberrant miR-139/Jun feedback loop in human gastric cancer. Biochim Biophys Acta 2015;1853:481-488.

48 Lopez-Bergami P, Lau E, Ronai Z: Emerging roles of ATF2 and the dynamic AP1 network in cancer. Nat Rev Cancer 2010;10:65-76.

49 Salameh A, Galvagni F, Anselmi F, De Clemente C, Orlandini M, Oliviero S: Growth factor stimulation induces cell survival by c-Jun. ATF2-dependent activation of Bcl-XL. J Biol Chem 2010;285:23096-23104.

50 Lindaman LL, Yeh DM, Xie C, Breen KM, Coss D: Phosphorylation of ATF2 and interaction with NFY induces c-Jun in the gonadotrope. Mol Cell Endocrinol 2013;365:316-326.

-51 Sun Q, Yogosawa S, Iizumi Y, Sakai T, Sowa Y: The alkaloid emetine sensitizes ovarian carcinoma cells to cisplatin through downregulation of bcl-xL. Int J Oncol 2015;46:389-394. 